\title{
Heinrich von Kleist und Franz Kafka: „Blutsbrüder" unter sich
}

Es ist nicht unwahrscheinlich, dass Kafka Kleist bereits während seines JuraStudiums las. So jedenfalls erinnerte sich der Schulfreund Hugo Bergmann. Dennoch gilt als sicher, dass eine ernsthafte Beschäftigung, die auch das Studium von weiteren Lebenszeugnissen des Preussen mit eingeschlossen haben könnte, nicht vor dem Jahreswechsel 1910/1911 eingesetzt haben kann. ${ }^{1}$ Dafür spricht ferner, dass der Name Kleist im Jahr 1911 gleich zwei Mal im Tagebuch des Pragers auftaucht. Der Ausdruck „Blutsbruder“, der sich eingebürgert hat und auch hier seiner Einprägsamkeit wegen vielfach verwendet wird, kommt so im Tagebuch Kafkas gar nicht vor. Sondern lediglich einmal die Bezeichnung „Bruder“ für Grillparzer. Da aber Kafka auch Indianergeschichten geschrieben und Gerstäcker gelesen hat, hat sich die „Blutsbruder“-Formel durchgesetzt; und dies bis hinein in die immer noch neueste, dreibändige, sich selbst als ultimativ verstehende Kafka-Biografie von Reiner Stach. ${ }^{2}$ Wen aber die Kafka-Forschung zum Kreis dieser „Blutsbrüder“ rechnet, das variiert. Kleist sowie Grillparzer und Kierkegaard zählen zum festen Stamm. Teilweise werden auch Flaubert und Dostojewski dazugerechnet. ${ }^{3}$ Kleist freilich zählt immer dazu; schliesslich war

\footnotetext{
${ }^{1}$ Dazu Hartmut Binders Kafka-Handbuch, Bd. I, S. 322. Ferner Peter-André Alts grundlegender Aufsatz zu Kleist und Kafka, in: Kleist-Jahrbuch 1995, S. 97-120.

${ }^{2}$ Diese Einschätzung findet sich in dem Band Die Jahre der Entscheidungen auf der Seite 410; freilich ohne, dass die Problematik der Begriffsprägung überhaupt erläutert würde.

${ }^{3}$ So in Hartmut Binders Kafka-Handbuch.

(C) Der/die Autor(en) 2021

B. Neumann, Umrisse einer Dritten Kultur im interdisziplinären Zusammenspiel zwischen Literatur und Naturwissenschaft, ELECTRISCHER PROMETHEUS. Umrisse einer Dritten Kultur im interdisziplinären Zusammenspiel zwischen Literatur und Naturwissenschaft, https://doi.org/10.1007/978-3-662-63204-8_5
} 
er es gewesen, der seinerzeit eigens nach Würzburg gereist war, den damals so viel beschrieenen „Mesmerismus“ näher kennen zu lernen. Also behauptete der Preusse seinen Platz auch dann, als Kafka den Kollegen Franz Grillparzer, den er im Jahr 1912 noch als positiv eingeschätzt und als „sehr männlich“ gelobt hatte, der eine altösterreichische Staatsbeamte kulant gegenüber dem anderen, ab 1916 dann, wesentlich im Zuge der eigenen Bemühungen, an die Weltkriegsfront zu gelangen, als blossen Büromenschen distanzierte. Søren Kierkegaard kommt dann im Jahr 1913 als weiterer „Freund“ ins Spiel, konnotiert mit dem Mozartschen Verführer-Thema und gestützt auf Kafkas eigene Vorliebe für das altösterreichische Musikgenie, auf dessen Don Giovanni (samt dessen Beliebtheit in Kopenhagen, Mozarts Witwe lebte nun dort) die Rede schon gekommen ist. In allen Fällen hatten beide „Blutsbrüder" mit unglücklichen Verlobungen und verhinderten Eheschliessungen zu kämpfen, kann bei ihnen eine nicht aufgelöste Vaterbindung diagnostiziert werden, galten sie insgesamt als „neurotisch“. Beide waren eben vom gleichen Indianerstamm. Folglich vermag die Kenntnis der Kleist'schen Werke und seiner „mesmeristischen“ Lebensproblematik bei Kafka mit Sicherheit angenommen werden, und zwar so früh, dass sie die gesamte, jahrelang sich erstreckende Schreibphase des Jugendromans umfasste. Folglich besitzt die Beschäftigung mit dem Thema Kleist-Kafka ihre unbezweifelbare Legitimität wie auch Tradition. Freilich muss gelten: „Die Vergleiche zwischen Kleist und Kafka, wie sie germanistische Arbeiten seit Jahrzehnten immer wieder unternommen haben, lebten meist aus der Sensibilität des unbefangenen Blicks, aber selten aus der Legitimität, die allein die historische Perspektive verschafft.“" Wenn damit die Forderung nach Standards eines „ideengeschichtlich differenzierenden Verfahrens" verbunden ist, ist dem nur zuzustimmen. Das gilt selbstverständlich auch und gerade für jedes transdisziplinäre Traktieren der Kafka-Kleist-Beziehung. Fraglos verband beide eine kunstbrüderliche Beziehung, erscheinen deren Filiationen in verblüffender Vielfalt, was zudem schon frühzeitig vermerkt wurde. Bereits am 6. VII. 1916 war im Berliner Tagblatt aus der Feder von niemand Geringerem als Oskar Walzel zu lesen gewesen, dass Kafkas Einleitungskapitel zum Verschollenen, eben Der Heizer, einschlägige Bezüge zur Prosa Kleists aufweise. Kurt Tucholsky hat dann nur wenig später Kafkas Strafkolonie ebenfalls als ein Kleist'sches Thema verstanden. Damit war ein Topos in der literarisch-literaturwissenschaftlichen Welt angekommen, der heutzutage bis zum (auch hier zitierten) Artikel André-Peter Alts reicht.

\footnotetext{
${ }^{4}$ Peter-André Alt, Kafka und Kleist, in: Kleist-Jahrbuch 1995, S. 97.
} 
Was es freilich bislang nicht gibt: Jenen Vergleich der beiden Autoren unter dem Aspekt des Mesmeristisch-Elektrischen, wie er im Folgenden unternommen wird. Der baut auf auf den offensichtlichen Gemeinsamkeiten beider Autoren: Als juristisch Ausgebildete beschreiben sie immer wieder „Rechtskonflikte, gestörte Familiengefüge, Täuschung und Selbsttäuschung, ... Schuld und Strafe, ... den Einbruch des Irrationalen in eine vermeintlich geordnete Welt der Normalität. Hinzu treten die auffälligen Parallelen der poetischen Formsprache und narrativen Komposition: die Charakterisierung der Figuren durch gestisch-mimischen Ausdruck, ... die Vorliebe für die Darstellung prägnant-novellistischer Situationen ..., die Tendenz zur einsinnigen Erzählchronologie, die Affinität zu personalem, weitgehend kommentarfreiem Berichtstil,... das Streben nach nüchterner, oft gefühlsarmer Diktion mit zahlreichen Anleihen bei amtlichem Kanzleiton und juristischer Fachsprache. ${ }^{\text {"5 }}$ Der Ausgangspunkt liegt dabei in jener besonderen Nähe zu Kleist, die zu besitzen Kafka seiner Dauerverlobten Felice am 2. XI. 1913 detailliert mitteilte. Daraus wurde dann, (aber nur in der Sekundärliteratur, nie bei Kafka selbst!) der hier so viel beschworene „Blutsbruder“ Heinrich von Kleist, wobei diese ,indianerhafte“ Bezeichnung keine wörtliche Quelle in all dem besitzt, was Kafka je über den Preussen geschrieben hat. Der „Blutsbruder“ romantisiert Kafka ein wenig in Richtung auf dessen Indianergeschichten und verhinderte die allzu direkte, historisch getreue, aber heutzutage politisch unkorrekte Berufung auf den ja dezidiert antifranzösischen Kleist mitsamt seines so grimmig-neurotischen Kampfes gegen Napoleon. Ein „Befreiungskrieg“, der zudem auch das Gebiet der elektrischen Nachrichtenübermittlung erfasste! Kleist unterstrich in seine Berliner Zeitungsartikeln die Kriegswichtigkeit derselben, was alles zusammen im Verschollenen seinen Niederschlag in der zutiefst negativen Delamarche-Figur mitsamt ihrem Deutschenhass gefunden hat, eine Auseinandersetzung im übrigen zwischen Liftboys in ihren elektrisch betriebenen

\footnotetext{
${ }^{5}$ Peter-André Alt, Kafka und Kleist, a. a. O., S. 98. Alts Artikel erbringt eine genaue und inspirierende Auflistung der Aspekte inhaltlicher Art, die Kafka und Kleist verbinden, ferner formale Gesichtspunkte, sehr überzeugende, die aber im Verlauf des Aufsatzes sich in die Erkenntnis aufzulösen drohen, dass beide Autoren halt anderen geistesgeschichtlichen Epochen angehören und dadurch verschieden bleiben. Offen bleibt eigentlich die (entscheidende) Frage: Was verbindet den „Modernen“ Kafka mit dem schroff tragischen, auf seine Art noch ,romantischen“ Kleist, worauf gründete die besondere Identität einer Dichter-Identifikation, für die die Bezeichnung „Blutsbrüderschaft“ akzeptiert zu werden vermochte?
} 
Aufzügen. Ferner gehört in diesen Zusammenhang auch ein Hinweis darauf, dass die oben zitierte, wie auch andere Mitteilungen an Felice, sehr oft darauf abzielten, der Frau die Lebens- und damit Eheuntauglichkeit ihres Prager Partners vor Augen zu stellen. Der durch Suizid geendete Kleist war dem jungen, noch dandyhaften Beamten Kafka da ein willkommenes Beispiel. Wenn Kafka brieflich schreibt, Kleist habe, als er sich ,im Gedränge äusserer und innerer Not am Wannsee erschoss, den richtigen Ausweg gefunden" "6 , so wird hierin eine eigene Lebensunfähigkeit unter- und ausgestellt, die wohl im Fall Kleists, aber derart stringent eben nicht in dem Kafkas gegeben war (der nie einen Selbstmordversuch unternommen, geschweige denn den Versuch, durch Schreiben zu überleben, aufgegeben hat, und der schliesslich durch Krankheit starb, Dank dem Freunde Klopstock unter Morphin-Einwirkung). Selbstmordgedanken mögen beiden gemeinsam gewesen sein, gehören ja zur Grundausstattung des modernen nervösen Intellektuellen. Die finale Tat aber gehört ganz ausschliesslich Heinrich von Kleist an, wie immer auch beide gehemmt und introvertiert (im Fall Kafkas: eher still, zurückhaltend), von Krankheit gezeichnet, am Leben verzweifelt und dem Schreiben verfallen gewesen sein mögen. Wobei aber, nicht zu vergessen, die Krankheit im Fall Kafkas keine zuerst psychische, sondern eine handfest physische gewesen ist. Gewiss hatten beide eine problematische Verbindung mit ihrer Familie; eine ,nicht normale“ Bindung an eine Lieblingsschwester; Vaterprobleme und daraus folgend eine äusserst komplizierte Verlobungsgeschichte gemeinsam. Ob man freilich bei solchem Durchgang durch beider gemeinsame Gebrechen nun auch noch Henriette Vogel, die krebskranke Sterbegefährtin Kleists, mit Dora Dymant gleichsetzen darf, nur weil letztere „kindlich“ gewesen sei, und zudem die „letzte Frau in Kafkas Leben“, steht dahin. „Erscheint Kleists Erkenntniskrise, die man gemeinhin mit dem Namen Kants verbindet, als singulärer Ausdruck persönlicher Zweifel an der Möglichkeit absoluter Wahrheit, so bleibt Kafkas negative Zeitdiagnostik in ihren Grundzügen epochenspezifisch, insofern sie eingebettet ist in den Kontext der Moderne und deren grundsätzliche Analyse von Entfremdung, Ich-Dissoziation und Sprachzerfall“".7 Das ist gewisslich wahr. Dennoch fragt man sich, ob Kleists Erkenntniskrise nicht eben eine exemplarische Zwischenstation auf dem Weg der Romantik in die Moderne gewesen ist. Zumal ein wesentlicher Unterschied zwischen den beiden „Blutsbrüdern" darin bestand, dass Kleist in erster Linie für die Bühne (und damit für

\footnotetext{
${ }^{6}$ F. Kafka, Briefe an Felice und andere Korrespondenz aus der Verlobungszeit, hrsg. von J. Born und E. Heller, Frankfurt/Main 1967, S. 460.

${ }^{7}$ P.-A. Alt, Kafka und Kleist, a. a. O., S. 101.
} 
eine Form adlig-bürgerlicher Öffentlichkeit) geschrieben hat; Kafka dagegen bereits einem solipsistischen Nacht-Schreiben verfallen war, das Öffentlichkeit kaum noch suchte. Stattdessen so etwas wie eine autoerotische Erfüllung im hoch „electrisch“ geladenen nächtlichen Schreibprozess fand. Wichtig ist gewiss, das Fehlen jenes ursprünglichen Aufklärungs-Optimismus bei Kafka herauszustellen, wie er andererseits Kleist noch durchaus gestreift hat. Für Kafka dagegen gilt: Sein Denken und Schreiben steht immer schon im entschieden desillusionierenden Licht des ausklingenden 19. Jahrhunderts als dem Zeitalter Darwins, Machs und Haeckels, Nietzsches und Kierkegaards. Es steht aber eben auch bereits am Beginn des Säkulums von Maxwell, Einstein und Heisenberg. Nichts ist bei diesem Prager Assimilanten noch als streng jüdisch Geprägtes zu betrachten. Der Talmud erscheint wesentlich entfernter, als jene besondere ,altösterreichische" Modernitätserfahrung um und nach 1900, wie sie sich, auch Alt weiss dies, „ebenso bei Hofmannsthal, Schnitzler, Thomas Mann und Rilke artikuliert." ${ }^{\text {8 }}$ Gemeinsam mag Kafka wie Kleist allerdings die Reflexion der in der Bibel dargestellten Sündenfall-Problematik gewesen sein. Doch während bei Kleist (im wesentlichen gegen seine eigene Dramen-Praxis) noch die Hoffnung auf Rekonstruktion des Verlorenen zugegen ist, deutlich etwa im Marionettentheater, kann und will sich Kafka die Wiederherstellung eines paradiesischen Zustands gar nicht mehr imaginieren. Bei ihm beginnt Menschheitsgeschichte eigentlich erst mit der Vertreibung aus dem Garten Eden. Auch schreibend ist dieser epochale Verlust bei ihm nicht mehr zu beheben, allenfalls ist er noch erträglich zu machen. Kleist ist hier überall entschiedener, tragischer, schroffer und weniger konziliant. Die zeitgenössisch verbreitete Sprachkritik etwa eines Hofmannsthal, dem die Wörter im Munde ,zerfielen“, war zweifellos bereits bei Kleist in Ansätzen vorgebildet. Eigentlich aber auch das nur theoretisch. Seine Dramen leben im Gegenteil von ihrer magisch-atemlos-labyrinthischen Sprachgewalt, als manische Kommunikation eines durch Schreibekstase vom Stottern Befreiten. Wie anders dann bei Franz Kafka, dem einsamen Nacht-Schreiber. Hier schreibt bereits einer, der qual- bis lustvoll seziert, was ihm das Wesentliche erscheint, gerade weil es ihm nicht zu glücken scheint: „Ich kann nicht schreiben ... Mein ganzer Körper warnt mich vor jedem Wort, ehe es sich von mir niederschreiben lässt, schaut sich zuerst nach allen Seiten um; die Sätze zerbrechen mir förmlich, ich sehe ihr Inneres und muss dann aber rasch aufhören. “9

\footnotetext{
${ }^{8}$ Peter-André Alt, Kafka und Kleist, a. a. O., S. 104.

${ }^{9}$ Franz Kafka, Briefe an Felice..., a. a. O., S. 85.
} 
In unserem Vergleichs-Zusammenhang ist noch hinzuzufügen das gemeinsame Interesse an Phänomenen des Mesmerismus, also am Phänomen des noch wissenschaftlich unbegriffenen, darum „electrischen“ Stroms als eine Art Lebenskraft mit verblüffender Fernwirkung. Hinzu gesellt sich insgesamt ein Verstricktsein in den Bereich des frühromantischen Elektrizitätsrausches, der sich bei Kafka freilich nur im Schreiben selbst, als seiner eigentlichen Lebenstätigkeit, konzentriert wiederfindet, während er bei Kleist sowie den Frühromantikern noch den Galvinismus umfasste, zuckende Froschschenkel neben glühenden Liebesergüsse. Die „dramatische“ Äusserung als tragische Dramenhandlung ist bei dem Prager nicht mehr zugegen, war freilich für Kleist noch zentral, und für die Frühromantiker jedenfalls erreichbar. Kafka dann ist „modern“ darin, dass er primär für sich selbst, und nicht mehr für die Bühne oder ein Lesepublikum schreibt. Folgerichtig hat er auch nur ein einziges Dramenfragment, im Stil des Maeterlinck'schen „lyrischen Dramas“ gehalten, hinterlassen. ${ }^{10}$ Im Gefolge von Schopenhauer und dann Kierkegaard sah der Prager Leben als heillose Verstrickung, jedenfalls als nicht erlösbares an. Der Apfel der Erkenntnis, vom mythischen Vater in den Rücken des Gregor Samsa versenkt, gerät in der Verwandlung zur Ursache eines quälenden Sterbens, eines Todes aus verrottendem Fleisch heraus; während die Frühromantiker noch auf die elektrische Erlösung der Puppe bzw. der Marionette setzten, was E.T.A. Hoffmann und Kleist miteinander verbindet. Und doch fehlt bei Kafka keineswegs, und das stellt ihn entschieden in's Gefolge der (früh)romantischen Hoffnung auf absolute, endliche erlösende Erkenntnis, der dominierende Zug ins Absolute und paradoxal Grenzüberschreitende hinein ganz und gar nicht. Im Gegenteil: Alles Streben bei ihm das bereits zitierte "stehende Marschieren“; und ein immerwährender vergeblicher „Sturmlauf gegen die Grenze“. Gewiss war der Prager vom Annalistischen her ein Kind des wissenschaftsgläubigen 19. Jahrhunderts. Und doch hat er schreiben können: „Alle Wissenschaft ist Methodik im Hinblick auf das Absolute. Deshalb ist keine Angst vor dem eindeutig Methodischen nötig. Es ist Hülse, aber nicht mehr als alles ausser dem Einen." 11

Das „Eine“ wiederum scheint in diesem Kontext jene Grenzüberschreitung zu sein, die all sein Schreiben anstreben wollte. Bei Kleist dagegen treffen wir

\footnotetext{
${ }^{10}$ Siehe dazu Peter Szondi, Das lyrische Drama des Fin de siècle, Frankfurt/Main 1975, S. $331 \mathrm{ff}$.

${ }^{11}$ Franz Kafka, Hochzeitsvorbereitungen auf dem Lande und andere Prosa aus dem Nachlass, hrsg. v. Max Brod, Frankfurt/Main 1976, Bd. 6 der Taschenbuchausgabe in sieben Bänden, S. 52.
} 
noch den Reflexionsbegriff im Zentrum des Nachdenkens an, auch darin ist er der, wenn man so will, verunglückte und durch Einsicht verunsicherte Schüler des grossen Immanuel Kant, dieses Auslösers seiner Lebenskrise. Ganz anders bei Kafka. Bei diesem tritt das Leben an die Stelle der Reflexion, der Prager ist darin schon der „Blutsbruder“ des Existenzphilosophen Kierkegaard. Den Tatbestand belegen am intensivsten jene Zürauer Aphorismen, wie sie sich Kafka in den Jahren 1917/1918 notiert hat. Das Bewusstsein des unabwendbar verlorenen Krieges bewirkte eine Rückwendung zur allein im Schreiben sich erfüllenden Existenz, die, mit Blick auf die biblische Erzählung vom Sündenfall, eben nicht mehr am Baum der Erkenntnis, wie noch bei Kleist, sondern ausdrücklich nur noch an dem des Lebens interessiert ist. (Was, nebenbei bemerkt, auch seinerseits das Kafka-Vorbild Goethe gewesen ist, der da, wo es ihm um Wissenschaft und Erkenntnis geht, in der bekannten Schülerszene des Faust, den „Baum des Lebens“ ausdrücklich „grün“ sein lässt, und den der Erkenntnis „grau wie alle Theorie"). Kafka vermochte also seinem Goethe in diesem zentralen Punkt treu zu bleiben (so wie auch in dessen Wendung gegen die Spektralanalyse des Lichts bei Newton, vermute ich). Denn was Maxwell und Einstein am Licht interessierte, dessen elektromagnetische Wellenbewegung und Geschwindigkeit, berührte sich gar nicht wesentlich mit Newtons Licht-Untersuchungen, eher im Gegenteil. „Den Ausgangspunkt für (Kafkas, B.N.) pessimistische Philosophie menschlicher Welterfahrung bildet ... die Deutung der Sündenfallerzählung, in deren Zentrum jedoch nicht die Frage nach der Erkenntnis, sondern jene nach dem paradiesischen Baum des Lebens steht, dessen Früchte ursprünglich Unsterblichkeit verleihen sollten. Im 82. Aphorismus der Betrachtungen heisst es: ,Warum klagen wir wegen des Sündenfalles? Nicht seinetwegen sind wir aus dem Paradiese vertrieben worden, sondern wegen des Baumes des Lebens, damit wir nicht von ihm essen." 12 Dem entspricht, wie gesagt, auch der Unterschied in Kafkas und Kleists Schreibpraxis: Wo Kleist sich noch mit dramatischtragischer Mitteilung an einen Zuschauer/Zuhörer wendet, in Dramen, auf der Bühne aufzuführen, wandert das „Wesentliche“ bei dem Modernen Kafka ganz „existentialistisch“ ins Innere des Schreibaktes selbst ab, der zudem noch dem Licht entzogen wird, um sich im Nachtschreiben zu erfüllen, wie er selbst es uns in seinem Bericht über die nächtliche Verfertigung des Urteils so beeindruckend detailliert überliefert hat.

\footnotetext{
${ }^{12}$ In dieser Stossrichtung zitiert bei Peter-André Alt, Kafka und Kleist, a. a. O., S. 111.
} 


\subsection{Jiu-Jitsu anstelle des antik-archaischen Zweikampfs? Fortschreibung eines elektrisierenden Motivs}

Die obenstehenden Überlegungen stellten so etwas wie eine ganz „moderne“ Form des intellektuellen Wettkampfs, ein argumentatives Kräftemessen dar; auch sie sozusagen ein Jiu-Jitsu-Kampf der Erkenntnis, weil nämlich der Schwung, die Energie des gegnerischen Arguments dazu diente, den Gegner zu Fall zu bringen. Von gleicher Struktur erscheint demnach jener intertextuelle Gruss, den der noch junge Autor Kafka an den da bereits weltberühmten, freilich seit einem runden Jahrhundert durch Selbstmord verstorbenen Autor der Penthesilea gesandt hat. Über diese intertextuelle Huldigung ist in der Kafka-Forschung bislang nicht gehandelt worden. Sie erscheint aber markant; zumal sie eine Antwort auf eben jene Frage nahelegt, die der so aufschlussreiche Artikel Alts denn doch nicht enthält: Was machte gerade Kleist so interessant für gerade den Kafka des Verschollenen? War es womöglich jene „elektrische“ (oder eben damals „electrische“) Anziehungskraft, die Menschen in Anziehung und Abstossung verbinden konnte, vom antiken Heldenkampf vor Trojas Mauern bis hin zum neuzeitlichen Jiu-Jitsu in dem schummrigen (weil noch nicht vom elektrischen Licht erleuchteten, das Haus wird zur Handlungszeit gerade erst elektrifiziert) Schlafzimmer des ansonsten so restlos modernen ,all American girl“ Klara? In Klaras perspektivisch bereits elektrisch erleuchtetem Haus wird andererseits geboten: Die modern-mondän- ,,amerikanische“, womöglich gar „,feministische“ Form des Ringkampfs, anstelle eines finster-mythisch-vormodernen Zweikampfs zwischen homerischem Held und skytischer Amazone. Man kann als Ausgangspunkt der folgenden intertextuellen Betrachtung festschreiben: Das am meisten irritierende, weil kompromissloseste, seinerseits skandalös bekannte und in jedem Fall am meisten „elektrisch geladene“ Theaterstück des genialen Dramatikers Heinrich von Kleist war zweifelsohne seine Penthesilea gewesen. Das Drama wurde 1808 geschrieben; aber dann erst 1876 in Berlin uraufgeführt. Es war gewiss nicht Kleists erfolgreichstes Theaterstück, wohl aber mit Abstand sein am meisten verstörendes. Von den mesmeristisch- „electrischen“ Kräften einer tief ambivalenten Libido durchwaltet, für die sich nicht nur „Küsse auf Bisse reimte“, sondern die Personen auf die jeweilige ,attrattiva“" (Goethes italienisch-lateinischer Ausdruck für Egmonts Strahlkraft) ihres Partners so fatal, eben weil unvernünftig-schicksalsgebunden reagieren, als handele es sich um ein galvanistisches Experiment. Selbst der tote Froschschenkel zuckt noch, führt durch Reflexe vorgeschriebene Bewegungen aus, wenn die galvanistische Elektrizität ihn ins Leben 
zurückzurufen scheint. Kleists archaisches Theaterstück war freilich in Wahrheit seiner Zeit voraus. Nicht nur der ambivalente Zusammenhang von Libido und Aggression wurde freudianisch vorweggenommen und dramatisch grell auf die Bühne gestellt, geradezu expressionistisch bereits. Auch wegen seiner stilistisch hochgesteilten, dabei ausgefeilten und äusserst expressiven Sprache galt das Drama lange als künstlerisch überzeugend, doch leider unaufführbar. Letzteres wegen seiner kaum darstellbaren Kriegsszenen zudem voll mit archaischen Grausamkeiten, und so vermochte das Schauspiel erst runde 70 Jahre nach seiner Niederschrift aufgeführt zu werden - und machte dann immer noch kräftig Skandal. Hinzu kam, dass Kleist nach dem Jahr 1870 (Deutsch-Französischer Krieg, Sieg Preussen-Deutschlands) einseitig-unterkomplex als antifranzösischer Dichter vereinnahmt zu werden vermochte, eben als der „preussische“ Autor des Prinzen von Homburg; eine Tatsache, die andererseits bei dem noch jungen Kafka auf (politisches) Interesse stiess, auf offenbar entschieden antifranzösisches sogar, horribile dictu. Wer nun eine Auseinandersetzung mit diesem Faktum suchte, wird von der Kafka-Forschung (und nicht nur der seit 1945 in Deutschland) enttäuscht werden. Doch alle Begleitumstände erscheinen eindeutig: Franz Kafka las damals Memoiren von Kriegsteilnehmern des DeutschFranzösischen Krieges, dessen Ausgang Preussen-Deutschland zur Weltmacht erhoben hatte, und zwar, sein Tagebuch belegt es, mit hoch positivem, gar identifikatorischem Interesse. Die 100-Jahr-Feier des Freiheitskämpfers Theodor Körner im frühen Film faszinierte und motivierte ihn, brachte ihn zu hektischem Nachtschreiben, wie dargestellt. Die Zeichnung des Franzosen Delamarche im Roman als eines rachsüchtigen, ressentimentgeladenen Intriganten rührt wesentlich daher und nimmt einen so breiten Raum ein, dass er der durchgehenden Vernachlässigung dieses Rache-Franzosen durch die Kafka-Forschung in etwa entspricht. ${ }^{13}$

Dieser Begründungszusammenhang kann noch ausgeweitet und vertieft werden. Seit den Anfängen im Jenaer Kreis war romantisches Dichten immer auch eine „Selbsterregungskunst“, ein Kampf gegen den Dämon des ennui und den des Nihilismus, gewesen. „Novalis empfiehlt die ,Gemütserregungskunst", seine Technik des Romantisierens, was im Kern bedeutet: sich hinaufstimmen.“ Und: „So aber empfanden sich die Romantiker: als absturzgefährdet, und das macht sie zu unseren Zeitgenossen. Das vormoderne Bewusstsein konnte

${ }^{13}$ Vgl. dazu die Belege in Bernd Neumann, Aporien der Assimilation, München 2007, S. $47 \mathrm{ff}$. Es ist bezeichnend, dass sich zu diesem Thema keine weitere Literatur anführen lässt. 
sich nicht vorstellen, aus der Welt zu fallen. Irgendein Jenseits gab es immer. Erst die Moderne sieht sich ohne metaphysischen Rückhalt mit der Endlichkeit konfrontiert, sie weiss sich nicht mehr selbstverständlich von einem sinngesättigten Kosmos getragen." 14 In Kleist wiederum fand die deutsche Romantik, nur war es nun nicht mehr die frühe Jenaer, sondern bereits die nationalbewusste in Berlin und vor allem in Heidelberg, ein rabiates Mittel gegen den bereits existentialistisch gefürchteten Absturz ins Nichts oder in die Langeweile. Dieser neu entfachte Nationalismus trat befeuert durch den Kampf gegen die Fremdherrschaft (des andererseits aber auch masslos bewunderten) Napoleon auf, dieser „Weltseele zu Pferde“ (Hegel), dem andererseits Beethoven seine „Erioca“Widmung wieder entziehen zu müssen vermeinte als der Schlachtenheld sich zum Kaiser krönte, und das auch noch eigenhändig. In Kafkas Tagebüchern bildet Napoleon einen fester Bestandteil; sein Russlandfeldzug wurde dem Prager Autor am Ende zum mythologischen Bild eigenen Scheiterns in den unendlichen Eiswüsten des eigenen Ichs, taumelnd am Rande des Tuberkulose-Todes, dieser ausgerechnet aus dem gefürchtet-verehrten Russland herübertönenden Sirene. Konkreter: Kafka sah seinen Wohnungswechsel nach Berlin, zusammen mit Dora Dymant durchgeführt, ein Stück weit ja durchaus als Parallele zu Napoleons so vergeblichem wie verderblichem Versuch, den europäischen Osten, die Weiten Russlands zu erobern. Seine Vorstellung vom (sozialen und kulturellen) Leben als Kampf verwies ihn ohnehin auf den Franzosen; was andererseits Napoleon für die Emanzipation der europäischen Juden getan hatte, wusste und schätzte er ebenfalls. Dennoch wohnte seiner Nähe zu Kleist notwendig auch eine Nähe zu Theodor Körners Freikorps-Kampf (wie gesagt: die Kino-Inspiration zum Verschollenen) und später dann gar zu Hugo von Hofmannsthals Konservativer Revolution ein. So verhielt es sich damals eben.

Was hier zu demonstrieren sein wird, denn das dezidiert antifranzösische und prohabsburgische Element in Kafkas Erstling darf nicht länger vermeintlicher politischer Korrektheit halber ausgeklammert bleiben. In den romantischen Kreisen Deutschlands hatte nach der Schlacht von Jena und Auerstädt ein Umschwung eingesetzt. Napoleon wurde immer mehr dämonisiert; geriet schliesslich zu einer Mischung aus Mephisto und Prometheus, als welcher er dann bei E.T.A. Hoffmann ,zur monumentalen Gestalt aus der Nachwelt des ,tierischen Magnetismus' (sich auswächst, B.N.), der damals als medizinische Praktik und als naturwissenschaftliche Spekulation die Zeitgenossen, die nach

\footnotetext{
${ }^{14}$ Rüdiger Safranski, Romantik, a. a. O. S. 314 und S. 316.
} 
Wundern und Geheimnissen verlangen, zu faszinieren beginnt. Napoleon also als grosser Magnetiseur, der einen ganzen Erdteil mit seinen magnetischen Strichen in Schlaf und Taumel versetzt, ein Gott des leeren Himmels und der neuen Zeit, in der es gilt: Die Politik ist das Schicksal." ${ }^{15}$ Die zuletzt zitierte Diagnose hatte, wie bekannt, Napoleon seiner Zeit gestellt; die reagierte darauf entsprechend lebhaft. Man hasste nun in dem einstmals Verehrten wahlweise den Despoten, den Revolutionär, den Geist des Machtzynismus oder den des antiklerikalen Rationalismus; die deutsche Öffentlichkeit kreierte in ihm summa summarum die Gestalt eines hybrid entfesselten Fichte'schen Ichs, dem die gesamte übrige Welt zum Nicht-Ich und Spielmaterial geraten sein sollte. In der Beförderung des Napoleon-Hassers Kleist zum „Blutsbruder“, und in der Anlage des Verschollenen insgesamt (Charakterisierung des Delamarche; Struktur des „Hotels Occidental" in seiner altösterreichisch-ungarischen Besatzung) übernimmt Kafka Erhebliches vom antifranzösischen, nationalistischen Geist einer nunmehr dominierenden Heidelberger Romantik, die dann ausgerechnet im Preussen Kleist ihre „Verzückungsspitze“ (Nietzsche) finden würde. Man muss das sehen, benennen und nicht mehr ängstlich ignorieren. Im Erstling Kafkas herrscht eben nicht ausschliesslich amerikanische Saloppheit, oder vorweggenommene Demonstration Foucault'scher Erkenntnisse, wie man zuweilen gemeint hat, sondern eher der originale Geist „Kakaniens“. Denn durch die immanenten Bezüge auf den brüderlichen Autor von Zerbrochenem Krug, Käthchen von Heilbronn und Pethesilea; durch Kafkas zeitgleiche Lektüre, und sogar durch seine damaligen Kino-Besuche war der Verschollene notwendigerweise, mit Massstäben heutiger ,political correctness“" gemessen, ,nationalistisch kontaminiert“.

Eine Rekonstruktion der bestimmenden Züge der „Heidelberger Romantik“, wie sie in gewissem Sinne in Kleists Dramawerk gipfelte, gilt es daher in diesem Zusammenhang anzugehen. Zwischen 1806 und 1815, nach dem Zusammenbruch Preussens, und in den Jahren der antinapoleonischen Kriege, gewann diese Romantik ihr scharfes politisches Profil. Es galt nun, sich der eigenen Vergangenheit zu versichern, und möglichst auch der Vorgeschichte der gesamten Menschheit, wie sie einst im Osten begonnen hatte. Das stand also damals zur Rede, wurde beispielsweise in Berlin von Friedrich Carl von Savigny angemahnt (auch er gehörte zum Heidelberger Kreis). „Man wird bald bemerken, ... dass die ignorierte Vergangenheit sich rächt, dass sie, wenn sie nicht bewusst ergriffen und fortgebildet wird, als blinder Zwang sich hinter dem Rücken der Akteure

\footnotetext{
${ }^{15}$ Rüdiger Safranski, Romantik, a. a. O., S. 285.
} 
durchsetzt, dass man also die erhoffte Zukunft nicht gewinnt, wenn man die Verbindung zur Vergangenheit zerstört."16 Zumal ja auch die griechische Antike ihrerseits inzwischen ein anderes Antlitz gewonnen hatte. Sie blickte nämlich, mit den Augen der Heidelberger Romantik angesehen, nicht mehr im klassischen Geiste Winckelmanns „still“, „edel“ und womöglich auch noch „einfältig“ zurück. Dieses Schaf, wenn es je eines gewesen war, war jedenfalls nunmehr zum Wolf geworden. Friedrich Schlegel beispielsweise hatte bereits in seinen frühen Schriften auf die orgiastischen, dionysischen Züge der mystischen hellenistischen Götterdienste hingewiesen. Novalis hatte, in seiner sanften Art, immerhin noch von einem „Geist der bacchantischen Wehmut“ geschrieben. Man realisierte nunmehr, dass nichts anderes als Sklaverei die reale Grundlage der griechischen Polis gebildet hatte. Auch, was dann Friedrich Nietzsche als entlaufener Pietist weiter ausfalten würde, dass die Griechen ihrerseits eigentlich skeptisch bis unglücklich gewesen seien. Und das gerade auch in der Blüte ihrer kunstgesalbten Freiheit, im grossen 6. Jahrhundert vor Christi! Der fremde Gott Dionysos gewann neue Bedeutung gerade auch im Umkreis von Schlegel, Schelling, Hölderlin und Hegel. „Die Freunde Hegel, Schelling und Hölderlin, hatten schon im Tübinger Stift einen privaten Kult mit ihm getrieben, und die dionysischen Mysterien der Wiedergeburt und Erneuerung hatten ihre Fantasie beschäftigt. Dionysos ist der Gott des Weines, der entfesselten Sinnlichkeit, der Lust, ein Gott aber auch, der zerrissen wird, stirbt, um wiederzukommen."17 Bereits Schopenhauer hatte solchen Einsichten nicht fern gestanden. Beim Lyriker Eichendorff wird es sich dann so verhalten, dass in seinen verwilderten, dämmerigen Parks die Marmorstatuen der Venus erneut zu unheimlichen Leben erwachen. Hölderlin wird am Ende von der, von ihm wiedererweckten, verzehrend-göttlichen Antike verschlungen werden. Alles steuerte derart auf die politische, patriotischnationalistische Wende und damit auf Kleist als das Genie ultimativen Hasses hin, den Vernichter des alten Reichs, den nunmehr dämonisierten Napoleon, seinerseits zu vernichten. Selbst der sanfte und naturwissenschaftlich beruhigte Novalis hatte ja mit Glauben und Liebe oder Der König und die Königin damals Aphorismen erscheinen lassen, in denen der preussische König Wilhelm III. samt seiner schönen Königin Louise gar eigenwillig-republikanisch, vor allem aber katholisch-umfassend verklärt wurden. Dies war auch eine Antwort auf Kants „preussischen“ Optimismus gewesen, der ja bekanntlich tatsächlich noch gemeint

\footnotetext{
${ }^{16}$ R. Safranski, Romantik, a. a. O., S. 243.

${ }^{17}$ R. Safranski, Romantik, a. a. O., S. 247.
} 
hatte, selbst ein Volk von Teufeln liesse sich republikanisch zähmen. So etwas glaubte die Heidelberger Romantik beileibe nicht mehr. Sie hatte den einen dominierenden Grund dafür, wie er auch in Kleists Wiener Verbindungen (und damit später auch in Kafkas Schreibbewusstsein bei Abfassung seines Jugendromans) zugegen gewesen ist: Das katholische Habsburg war der einzige Gegner Napoleons, der ungeschlagen geblieben war,Vorläufer des endlich umfänglich siegreichen Russland. „Die anfänglich revolutionäre, republikanische Begeisterung geht bei Novalis, wie später auch bei Schlegel, in religiöse Ordnungsvorstellungen über. In dem Augenblick, als das alte Reich unterging, verklärten sie es zum Mythos, und es wurde daraus die Vision eines friedlichen Zusammenlebens der Völker unter dem Schirm einer christlichen Schutzmacht, deren Mandat nicht auf das protestantische Preussen übergehen sollte, sondern beim katholischen Habsburg zu bleiben hätte“ - und damit bei Kafkas Staat, dem sich dieser als Beamter in der tiefen Loyalität des assimilierten Juden verpflichtet fühlte, im Gefolge der Judenemanzipation durch den aufgeklärten Joseph II. „Warum Habsburg? Und nicht Preussen? Weil ihnen Habsburg als Bewahrer der Reichsidee und darum als übernationale Macht erschien. Sie ahnten das Unheil, das der entfesselte Nationalismus bringen würde. Sie träumten von einem Reich, das mehr sein sollte als ein zum Imperium erweiterter Nationalstaat. Vertraut mit der mittelalterlichen Geschichte, kannten sie jene mit dem Reichsmythos verbundene Tradition, für die das Imperium Romanum und seine Fortsetzung als Imperium Christianum das vom Buch Daniel prophezeite vierte Weltreich darstellt, mit dessen Zusammenbruch die Welt untergehen würde, weshalb diesem Reich aus endzeitlicher Sicht die Rolle des ,katechon' zufiel, die Kraft, die dem zweiten Thessalonicher-Brief des Paulus zufolge den Widersacher Christi und damit den Weltuntergang noch aufhalten konnte. Realpolitisch gedacht war das natürlich nicht, ..."18 - aber so ist es eben auf Kafka überkommen! Insofern rekonstruiert das obige Zitat, darin (und durch seine schöne Sprache ohnehin) in ganzer Länge gerechtfertigt, eine wesentliche Facette von Kafkas Geschichtsbewusstsein bei Abfassung des Roman-Erstlings mit seinen langen ,altösterreichischen“ Passagen vor allem in der „Hotel Occidental“-Handlung, die ja ihrerseits erfüllt erscheinen von wenig anderem als der intensiv widerstreitenden Einheitlichkeit des von Maria Theresia gegen den siegreichen preussischen Friedrich II. gebildeten Zusammenschlusses Wiens mit Budapest/Ungarn. Hinzu kam, dass es ausschliesslich das Habsburgische Österreich war, das in mannigfachen

\footnotetext{
${ }^{18}$ R. Safranski, Romantik, a. a. O., S. 263.
} 
Formen den Kampf gegen Napoleon fortsetzte. Man kämpfte damals (der Tiroler Andreas Hofer) u. a. einen Guerillakampf nach spanischem Vorbild, und das auch noch in folgender Situation: Der leitende preussische Minister Stein hatte 1807/1808 noch mit Napoleon zu kooperieren versucht. Das scheiterte enttäuschend. Zusammen mit Gneisenau unternahm der Mann es danach, den Widerstand nun auch militärisch zu organisieren. Nahm das bislang Undenkbare in Angriff: Die Volkserhebung. Sie war bei ihm klugerweise gestützt von tiefgreifenden Sozialreformen vor allem für die ländliche Bevölkerung. Napoleon blieb dennoch der Stärkere. Der Protestant Stein flüchtete sich danach ausgerechnet nach Böhmen, in die Nähe von Prag, auf das Gebiet des katholischen Habsburg, wirkte dort als Symbol des umfassenden Widerstands gegen Napoleons Fremdherrschaft. Damit hatte die Stunde der politischen Romantik geschlagen, und war auch die Kleists ganz nahe gerückt. Der hatte, wie immer Preusse, aber eben auch der Gefährte von Adam Müller, immer schon die Nähe der Wiener Politik gesucht. Das erschien jetzt nur noch aktueller, man wollte zurück zu jener Ordnung, wie sie vor der Französischen Revolution bestanden hatte: Garantiert durch ein christlich geprägtes Weltganzes, das sich in seinen festen Bahnverläufen in Politik und sozialem Leben manifestieren sollte. Novalis hatte bereits 1798 geschrieben: „,... und wir fühlen uns von einer friedlichen Welt angezogen, wo eine Zentralsonne den Reigen führt, und man lieber Planet wird, als einen zerstörerischen Kampf um den Vortanz mitkämpft."19 Eine Welt, von der selbst noch der durch die moderne Atomphysik verschreckte Wilhelm Dilthey träumen wird, wie geschildert. Darauf besann sich die Heidelberger Romantik, wie ebenfalls bereits geschildert. Man lebte ja unter Napoleon in einer waffenstarrenden Welt, die Franzosen hatten als „Grand Nation“ ganz Europa überflutet, man hatte sich dagegen nun auch denkerisch-gefühlsmässig zu organisieren. Da lag der von Schiller wie Goethe promovierte Gedanke an eine zwar verspätete, aber dann alle anderen überholende „Deutschen Kulturnation" nahe, die sich selbst allerdings hochschätzen (aber deshalb andere nicht abwerten) sollte. Novalis schrieb damals: ,Jedes Volk/Hat seinen Tag in der Geschichte, doch/Der Tag des Deutschen ist die Ernte der/Ganzen Zeit.“ Das war hoch gedacht; man konnte nicht ahnen, dass diese Kulturvergötterung sich später einmal würde gebrauchen lassen für die Zwecke der Barbarei - wie immer diese auch bereits auf der Lauer lag etwa in Fichtes Reden an die Deutsche Nation aus den Jahren

\footnotetext{
${ }^{19}$ Zitiert bei Rüdiger Safranski, Romantik, a. a. O., S. 264.
} 
1807/1808. Der kosmopolitisch-universalistische Ansatz der Frühromantik jedenfalls verschwindet. Wird ersetzt durch die Beschäftigung mit dem „Deutschen“ in Märchen und Lied, Volkstum und „Blut“. Heidelberg samt seiner Schloss-Ruine wurde zwischen 1806 und 1808 zum Hauptquartier einer nunmehr auf Märchen, Mythen, Volkslieder und historische Überlieferung ausgerichteten Hochromantik, die das Erbe der Frühromantik mehr und mehr vergass oder nationalistisch umformte.

Noch aber war eher der „Philister“ der Hauptfeind, als der ethnisch Fremde. Die Brüder Grimm haben den Stil ihrer Märchen eher erfunden als gefunden. Doch im Gefolge der nunmehrigen „Volkserhebung“ gegen Napoleon wird jetzt umgekehrt die politische Propaganda entdeckt und facettenreich entwickelt. Szenen eines Kriegsausbruchs oder Bilder aus einem anderen „Vormärz“: Als am 28. März 1913 der Krieg gegen Napoleon offiziell erklärt wird, steht Schleiermacher in Berlin auf der Kanzel; es geht nicht mehr um Hermeneutik oder Henriette, sondern darum, das Publikum auf den Heldentod einzuschwören, während draussen schon die Pferde grasen und die Gewehre geladen an der Kirchenwand lehnen. Die gesamte Berliner Universität steht in Waffen, in mehr oder weniger spektakulären. Goethes Briefpartnerin Bettina von Arnim hat beschrieben, was da so alles vor sich ging: "Auch war es seltsam anzusehen, wie bekannte Leute und Freunde mit allen Arten von Waffen zu jeder Stunde über die Strasse liefen ... der Philosoph Fichte mit einem eisernen Schild und langen Dolch, der Philologe Wolf mit seiner langen Nase hatte einen Tiroler Gürtel mit Pistolen, Messern aller Art und Streitäxten angefüllt ... bei Arnims Kompagnie fand sich jedesmal ein Trupp junger Frauenzimmer, die fanden, dass das Militärwesen ihm von vorn und hinten gut anstand..." ${ }^{20}$ Sie alle zogen ins Feld gegen den grossen Magnetiseur, als der Napoleon bei E.T.A. Hoffmann aufgefasst worden war. Und hier kommt nun Kleists, die Kafka'sche Identifikationsfigur, ins Spiel. Safranski hat schreiben können: „Als einer der grössten Hasser zeigt sich Heinrich von Kleist. Man rechnet ihn gewöhnlich nicht zu den Romantikern. Aber legt man die Definition Carl Schmitts zugrunde, wonach Romantiker Leute sind, welche die jeweilige Wirklichkeit - occasionalistisch - zum Anlass nehmen, um ihr eigenes Ich imaginär zu entfesseln, dann war Kleist, insbesondere in jenen politisch erregten Zeiten, mit dem Extremismus seiner Gefühle und dem Absolutismus seines Ichs einer der genialen Romantiker. Aber auch ein

\footnotetext{
${ }^{20}$ Bettina von Arnim, Werke und Briefe, hrsg. v. Johannes Müller, Frechet 1961, Bd. 5, hier zitiert nach Rüdiger Safranski, Romantik a. a. O., S. 282.
} 
gefährlicher Romantiker.“21 Der Mann forderte zu Vernichtungskrieg und wollüstigem Totschlagen auf, seine Hermannsschlacht zeugt ebenso davon, wie seine Zeitungsartikel und zahlreichen anderweitigen Appelle an den teutonischen Hass seiner Landsleute. Als solcher ist dieser Blutsverwandte Kafkas in den kräftigen, seinerseits flammend rot gefärbten und rabiat antifranzösischen Strang im Verschollenen eingegangen, der nicht dadurch verschwindet, dass man ihn ignoriert. In diesem Zusammenhang ist nicht nur Kleists Ode Germania an ihre Kinder mit der Aufforderung „Schlagt ihn tot! Das Weltgericht/Fragt Euch nach den Gründen nicht!“ zu erwähnen; sondern eben auch des Dramatikers bedeutende Pentesilea, wo in der Schlussszene die Amazonenkönigin den fremden Griechen Achill, wie immer, nein: weil! sie ihm brünstig verfallen ist, mit ihren eigenen Zähnen zerfleischt und die Hunde auf ihn hetzt. Solch hoch heikle Innigkeit steht in starkem Kontrast dazu, dass Kleist - und das wiederum verbindet ihn tatsächlich mit seinem Prager „Blutsbruder“ - von seiner Braut Wilhelmine von Zenge erwartete, dass beide, selbst wenn sie im selben Haus zusammen lebten, jeden Tag einander Briefe zu schreiben hätten. So wie es dann auch Kafka mit Felice hielt, auch wenn die in der Regel weiter von ihm entfernt lebte. Kafka und Kleist: Wahlverwandte in der Form der Kommunikation, Geschwister im Geist der Ehelosigkeit um des Schreibens willen, produktive, vatergeschädigte Neurotiker des Patriotismus, - und beide grosse Meister der Novellenform mit ihrem Gebot des abrupten Umschlags, bei Boccaccio praktiziert und bei Paul Heyse dann zur nobelpreisgekrönten Regel erhoben! Dennoch war Kafka jener Autor, der niemals die Gattungsbezeichnung „Novelle“ für seine Texten in Anspruch nahm. Darüber, dass in seinen Traum-Erzählungen ein „Falke“ zu finden sei, hätte er wohl nur den Kopf geschüttelt, leise lächelnd, müde vom Schreiben. Hätte es sich um den Golem oder die Unerreichbarkeit der Lichtgeschwindigkeit gehandelt, die Sache hätte sich womöglich anders dargestellt. Davon später, wir müssen erst noch Klaras nicht-elektrifiziertes Schlafzimmer aufsuchen.

${ }^{21}$ Rüdiger Safranski, Romantik, a. a. O., S. 285 f. 


\subsection{Die Adaption der Kleist'schen Penthesilea in Kafkas Erstlingsroman}

Des Preussen Bernd Wilhelm Heinrich von Kleist Penthesilea war inspiriert gewesen von gleich drei antiken Tragödien des Euripides, von Medea, Hippolytos und den Bakchen; auch daher rührt, was Günter Blöcker ihre ,dionysische Verbalmusik“ hat nennen können. War sie bei ihrer Niederschrift noch zukunftsweisend gewesen, so hatte das Wien des Vorkriegs ihre besondere und durchaus problematische Modernität seinerseits, um 1900 herum, durchaus eingeholt. Bereits Friedrich Schlegel hatte schliesslich in seiner Abhandlung Über das Studium der griechischen Poesie geahnt und ausgesprochen, dass das besondere Interesse der frühen Romantik an extremen Gegensätzen nachdrücklich literarische Schule machen würde. Achill in Heinrich von Kleists Stück nennt sein feindliches Liebesobjekt ,halb Furie, halb Grazie“ (21. Auftritt). Noch Hugo von Hofmannsthal als der bedeutende Vertreter des Modernsten im Wien der Jahrhundertwende wird, in der Elektra, der besonderen Neigung seiner Zeit zum Schockierenden und Grässlichen seinen Tribut zollen. Hatte es ja auch schon in der Salomé getan in gleichermassen geglücktem Zusammenspiel mit einer Musik von Richard Strauss, die zwar nicht atonal heissen, aber doch von zeitgenössischer Atonalität inspiriert erscheinen konnte. Auch Kleists Schauspiel hatte Kafkas besondere Aufmerksamkeit durch die darin exekutierte zeitgenössische Tendenz geweckt, die Rezeption antiker Mythen vom hergebrachten klassischen Bild des ,edlen“ und „harmonischen“ Hellenentums abzusetzen. Wenn also bei Kleist die skytische Amazonenkönigin den homerischen Achill auch deshalb manisch suchte, weil dieser durch das barbarische Im-Staub-Dahinschleifen des toten Hektor einmal um das belagerte Troja herum, herostratischen Ruhm erlangt hatte, erfolgt bei Kafka die erste Bekanntschaft der Kontrahenten wie folgt: Der Begegnungs-Raum ist verdunkelt durch Baumwipfel, also ohne jedes Mondlicht (während draussen voschriftsmässig der Mond aufgegangen ist), und aus diesem Grunde vermisst Kafkas Protagonist schmerzlich ausgerechnet jene ,elektrische Taschenlampe, die er vom Onkel geschenkt bekommen hatte.“ „Klara kam eilends herein ... rief: ,Also wollen Sie mit mir kommen oder nicht?“ und stiess ihn mit Absicht oder bloss in der Erregung derartig an die Brust", dass der brave Deutsche aus dem Fenster zu stürzen drohte. ,,Jetzt wäre ich bald herausgefallen' sagte er vorwurfsvoll. ,Schade dass es nicht geschehen ist"... Und wirklich umfasste sie ihn und trug ihn, der verblüfft sich zuerst schwer zu machen 
vergass, mit ihrem vom Sport gestählten Körper fast bis zum Fenster.“22 Das sind, wenn schon nicht "Szenen einer Ehe“, so doch Szenen von einer, wie immer auch leicht ironisch gebrochenen, Kleist'schen Gewaltsamkeit. Als Karl Clara dann, wie vor ihm Achill die Amazonenkönigin, überwältigt und auf's Kanapee mehr wirft als ablegt, begann sie ihn ,so stark zu würgen, dass Karl ganz unfähig war, etwas anderes zu tun, als Luft zu schnappen." Der arme Knabe! Zumal auch diese neue Penthesilea ihre Niederlage souverän verdrängen wird. Wird sich freilich danach ihrerseits der (Mozart!)Musik ergeben, wobei das Bestialische neben dem ganz Zarten zu stehen kommt, ganz so, wie es Kafkas fin de siècle-Zeit gebot, und das eben nicht nur in Wien. Man hatte bereits in den Frühzeiten der Romantik damit begonnen, das wahres Wesen der Hellenen auch in Hysterie und lyrisch überformter Barbarität zu suchen, was sich ja mit „,moderner“ seelischer Differenziertheit im anbrechenden Zeitalter Sigmund Freuds bestens vertrug. Zumal der unheilbar tragische Riss im Sinngefüge der Welt, Kleists Hauptthema, auch in Wien, etwa in Franz Grillparzers Des Meeres und der Liebe Wellen, bereits zugegen erschien; zusammen mit des Hofrats unübersehbarer Vorliebe, im heraufbeschworenen mythischen Bild die tragisch-unheilbare Antinomie ihrerseits in der Götterwelt wiederzufinden. Insofern befand sich Kafka nahezu auf heimischem Terrain; war Prag gar nicht so weit von Wien angesiedelt, wenn der Prager unter Kleists Stücken gerade die Penthesilea favorisierte. Hinzu kam die besondere Art und Weise, jener selbst „hysterische“ oder, wenn man so will, „galvanistische“ Schlag, den der Kinogänger Kafka empfing, als er, in der Niederschrift des den Verschollenen einleitenden Heizers begriffen, in einem Prager Lichtspielhaus den Jubiläums-Film über den antifranzösischen Freiheitskämpfer Theodor Körner sich ansah, wie bereits erwähnt und übrigens unter Tränenfluss geschehen. Alle Romanhelden Kafkas sind von ihrem Autor als "Schuldige“ bezeichnet worden, worin beschlossen lag, dass sie als Juden gedacht wurden. Karl dagegen erscheint, wiederum nach Kafkas eigener Aussage, so konsequent emanzipiert bzw. assimiliert, dass er, als einziger Romanheld, ein Nicht-Jude und mithin kein „Schuldiger“ sein konnte. Dieser Karl nun wird, in New York an Land gegangen, seinerseits ganz konsequent dem mythischen amerikanischen Muster des Nach-Westen-Gehens folgen - jener amerikanischen Western-Mythe, die, noch in den siebziger Jahren des vergangenen Jahrhunderts, auch den (ebenfalls österreichischen) Schriftsteller (und nun Nobelpreisträger

\footnotetext{
${ }^{22}$ Franz Kafka, Der Verschollene in der Kritischen Ausgabe der Gesammelten Werke von Hans-Gerd Koch, a. a. O., S. 72 (siehe Anmerkung 38).
} 
von 2019) Peter Handke in seinem Langen Brief beschäftigt hat. Kafkas „Vorlage“, Arthur Holitschers Amerikareise-Bericht, hatte 1912 noch ausführlich das Gegen-Muster dazu herausgestellt: „Ich nehme mir's vor, in Amerika zuzusehen, wie dieses Volk sein Leben fristet. Es kolonisiert nicht. Es geht nicht nach dem Westen. Es bleibt in New-York sitzen, hockt lieber in Schmutz und Not beisammen, als irgendwo in der frischen Luft zu leben, wo's noch kein Ghetto gibt. “23 Holitscher selbst war, ganz wie Kafka, ein österreichisch assimilierter Jude. Kafkas Rossmann wird dann, wie immer nicht ganz freiwillig, seinerseits keineswegs in New York sitzen bleiben. Doch er wird bei allem „Kolonisieren“ dennoch nicht $\mathrm{zu}$ dem gelangen, was noch Holitscher als sein literarischer Cicerone versprochen hatte: Zum richtigen „Leben“ (das bei Holitscher nicht zufällig im Sperrdruck in den Text gesetzt wurde).

So weit, so einsichtig. Doch worin beweist sich des Knaben Tauglichkeit, die Karls Zutritt zur Neuen Welt erst ermöglicht? Zuerst muss in der Heizer-Episode (Kafka selbst schätzte sie als Bestes seines gesamten Romans) Karls soziale Mannbarkeitsprobe bestanden werden. Deren Besonderheit wiederum versteht nur, wer die Entstehungszeit des Heizers samt ihrer Hauptdiskurse näher betrachtet. Franziska Schössler hat dies getan. „Es sind im ,Verschollenen' zwei Traditionslinien, die den Text über das amerikanische Exil palimpsestisch grundieren: Zum einen werden biographische Details von Autoren eingearbeitet, insbesondere von Goethe und dem Freiheitskämpfer und Dichter Körner, so dass das frühe Fragment Kafkas als versteckte Künstlerbiographie gelesen werden kann. Karl ist ein Künstler, obgleich, oberflächlich betrachtet, seine Kunstbemühungen banalisiert oder gar dementiert werden ... Die andere Traditionslinie, die ebenfalls im Zeichen einer Künstlerinitiation eingearbeitet wird, ist eine mythologische; in Kafkas Romanfragment werden Mythen wie die des Prometheus und des Musenpferds Pegasos aufgenommen, zu ironischen Details verdichtet. “24 Hinzuzufügen wäre dieser Aufzählung mit ihrer einsichtigen Prometheus-Nennung nunmehr noch der Penthesilea-Mythos, der, wie angesprochen auf intertextuelle Weise, eben durch Kafkas impliziten Verweis auf den Text des „Blutsbruders“, in seinen Roman gelangt ist. Beide wiederum kommen, ausgerechnet, in Kafkas Karl als der unverkennbaren KinoVariation des berittenen Franzosenfressers Karl Theodor Körner zusammen. Ein

\footnotetext{
${ }^{23}$ Arthur Holitscher, Amerika. Heute und Morgen..., a. a. O., S. 20 f.

${ }^{24}$ Franziska Schössler: Verborgene Künstlerkonzepte in Kafkas Romanfragment „Der Verschollene“. In: Hofmannsthal-Jahrbuch, Bd. 6 (1998), S. 282.
} 
Tatbestand, aus dem Kafkas Namensgebung „Karl Rossmann“ allerdings abgeleitet werden kann? Im Einzelnen lässt sich hierzu biografisch rekonstruieren: Während Kafka bereits am 11. September die Eingangs-Szenerie des New Yorker Hafens träumend antizipiert, ist er immer noch mit dem zentralen literarischen Ereignis dieser Tage: Der Niederschrift des Urteils, fieberhaft zugange. Am 23. September ist ihm dann dieser schriftstellerische „Durchbruch“ (so der Kafka-Biograph Reiner Stach), also die literarische Verarbeitung seiner Begegnung mit Felice Bauer, gelungen. Immer noch im Produktionsrausch, mit den Anfängen des Heizers bereits befasst, verweigert der nächtliche Lustschreiber sich dennoch, nach dem orgiastischen Erfolg der Urteils-Niederschrift, jede weitere kurzfristige Befriedigung. Der Trieb soll gestaut werden, zumal alle bisherigen Anläufe zum Schreiben des Verschollenen, die laut Hartmut Binder bis auf das Jahr 1898 zurückgehen, endgültig im Juli 1912 verworfen worden waren. ${ }^{25}$ Kafka wartete nun $\mathrm{zu}$, verweigerte sich seiner durchaus anhaltenden Schreiblust. „Wie Odysseus widersteht er den lockenden Sirenen“26, und geht (wie dann später auch Handkes Held im Langen Brief) stattdessen in die allerneueste Mythenfabrik: In eines der ersten in Prag, im dortigen Landestheater, eingerichteten Kinos. Das war bei ihm nicht ungewöhnlich. In Kafka steht vielmehr einer der ersten regelmässigen Kinogänger der deutschsprachigen Literatur vor uns; er ist auch darin höchst „modern“. Freilich sah der Prager die Vorstellung damals keineswegs unter dem ultimativen Gebot einer „Faszination durch das neue Medium”, sondern musste sich eben auch vom Schreiben gewaltsam ,wegreissen" (Tagebuch 25. September 1912). Andererseits wird die Kinovorstellung allerdings bewirken, dass Kafka in fast manisch gesteigerter Schreiblaune heimkehrte. ${ }^{27}$ Der Prager hat folglich am Abend des 25. September ein Lichtspiel gesehen, das seinerseits den Verschollenen prägte. Und zwar mit Sicherheit mehr als alle „mit der Niederschrift des Urteils zusammenhängenden Vorgänge.“28 $\mathrm{Ob}$ Kafka wirklich noch im Geiste bei Felice gewesen sein konnte, nachdem er die ihr gewidmete Erzählung abgeschlossen hatte, scheint schon in sich zweifelhaft. Zumal er seine mehrfache Verlobte mit Sicherheit weder als Amazonenkönigin, noch als Jiu-Jitsu-Kämpferin sich jemals imaginiert hat. Gewiss ist andererseits,

\footnotetext{
${ }^{25}$ Hartmut Binder: Kafka-Kommentar zu den Romanen, Rezensionen, Aphorismen und zum ,Brief an den Vater', München 1976, S. 54 und 58.

${ }^{26}$ Hanns Zischler: Kafka geht ins Kino, Reinbek 1996, S. 86.

${ }^{27}$ Vgl. Peter-André Alt, Franz Kafka. Der ewige Sohn, München 2005, S. 215.

${ }^{28}$ Hartmut Binder: Kafka-Kommentar..., a. a. O., S. 59.
} 
dass Kafka die Niederschrift der neuesten uns bekannten Fassung des Heizers (als des Eingangskapitels des Verschollenen) unmittelbar nach dem Kinobesuch in Angriff genommen hat. Warum aber konnte ausgerechnet Karl Theodor Körner Kafka so begeistern, ihn, der nach dem Zeugnis Max Brods in seiner damaligen Frühzeit „von Nietzsche her" kam, und daneben bereits von „Goethe und Flaubert" dominiert wurde? ${ }^{29}$

Zur Beantwortung dieser Zentralfrage ist eine genaue Rekonstruktion des fraglichen Abends nötig. Ausweislich seines Tagebuchs erblickte Kafka aus seiner „Loge“ ein Programm von drei Filmen, wobei der erste - „Fräulein O., welche einmal ein Geistlicher verfolgte. Sie kam ganz nass von Angstschweiß nach Haus“ - zu Stücken wie „Seltsame Insekten“ und/oder „Die Insel Ceylon“ benachbart gewesen sein muss. Vorausgesetzt, die Programmabfolge, die Kafka erblickte, hat der Annonce in der Bohemia vom 25. September 1912 entsprochen - was höchst wahrscheinlich erscheint. In Bezug auf Körner wurden die ,wichtigsten und spannendsten Momente seines Wirkens“ versprochen. ${ }^{30}$ Den Abschluss machte dabei „Zur Erinnerung an den Geburtstag Theodor Körners: Theodor Körner. Sein Leben und Dichten - Aus der Jugendzeit. Der Student - Der Theaterdichter und seine Braut - Der Freiheitskämpfer.“31 Kafkas Tagebucheintragung wiederum fand die mit Abstand meisten Worte für den patriotischen Dichterfilm: „Körners Leben. Die Pferde. Das weiße Pferd. Der Pulverrauch. Lützows wilde Jagd." ${ }^{2} 2$ Die in unseren Tagen durchgesetzt ironische Kommentierung des patriotisch-heldischen Filmstreifens vermag zwar eine politisch korrekte Gesinnung zu signalisieren. Sie kann aber nicht mehr verhindern, dass der deutschnationale Streifen Franz Kafka damals sehr nachhaltig beeindruckt hat. Denn, philologisch-verlässlich rekonstruiert, steht eines fest: „Kafkas Niederschrift der Zweitfassung setzt mitten in Tagebuchnotizen ein“ - und zwar unmittelbar nach, ein erneutes Zitat: „Lützows wilde Jagd“ ${ }^{33}$ Mit anderen Worten: Jener Kafka, der damals, womöglich schon am Nachmittag des 25. September (denn die Vorstellungen begannen um 14,00 Uhr, um 16,30 Uhr,

\footnotetext{
${ }^{29}$ Max Brod: Streitbares Leben, München/Berlin 1969, S. 160 und 189.

${ }^{30}$ Vgl. Hartmut Binder, Kafka-Handbuch, a. a. O., Bd. 1, S. 434.

${ }^{31}$ Nach Hanns Zischler: Kafka geht ins Kino, a. a. O., S. 86.

${ }^{32}$ Tagebucheintrag vom 25. September 1912.

${ }^{33}$ Hartmut Binder: Kafka-Kommentar, a. a. O., S. 60. Peter-André Alts begründungslose Nennung des 26. September (in: Franz, Kafka. Der ewige Sohn, a. a. O., S. 344) überzeugt nicht.
} 
dann wiederum um 19,30 Uhr, und sie dauerten jeweils $90 \mathrm{~min})^{34}$, aus dem Kino kam, nahm spontan und begeistert die Niederschrift des Heizers in Angriff. Der Text wird dann am 2. Oktober fertig sein. Kafka las Brod am 6. Oktober daraus vor. Die entfesselt-kaskadenartig fließende Produktion des Heizers erschütterte Kafka dabei so sehr, dass er in Weinkrämpfe geriet, von denen er fürchtete, dass sie seine Eltern wecken könnten, wie er an Felice berichtete. Also: Karl Rossmann geriet Franz Kafka nach dem schimmelreitenden Filmhelden Karl Theodor Körner zu einem „Karl“, zu einem dichterischen „Rossmann“ auf einem in die USA versetzten Pegasos - wo das famose Pferd aber nur noch sehr wenig Nahrung finden wird. Selten wurde dieser Tatbestand wahrgenommen und als der dominierende Schreibanstoß realisiert, als der er allerdings gelten muss. Kafka selbst hat später in einem Brief an Felice unterstrichen, dass nur das HeizerKapitel aus ,innerer Wahrheit“ herkomme, der Rest aber in „Erinnerung an ein großes aber durchaus abwesendes Gefühl hingeschrieben" sei. ${ }^{35}$

Soweit die selbst „electrisch“-lichtspielhafte Genese der Kafka'schen Niederschrift erst des Heizers, dann des gesamten Verschollenen. Die Parallelität Amazonenkampf-Jiu-Jitsu drängt sich geradezu auf, wenn man Karl als den „Helden“ des Kafka'schen Romans an die Stelle der Heldin in Kleists Tragödie setzt. Wenn man so will: Der aus Prag abgeschobene Karl gelangt so neu und fremd nach Amerika, wie Penthesileas Amazonen rein zufällig auf die Griechen stiessen, die damals Troja belagerten. Das unverhoffte Zusammentreffen wirft beide aus ihrer Bahn. Die Amazone verliert ihren Königinnenstatus samt Vernunft und Lebenssinn. Karl verliert seine vorgezeichnete Karriere in Amerika. Gerät in eine tragische, durch Klaras fatale Anziehungskraft bewirkte Wiederholung des Ausgangspunktes seines Schicksals: Unschuldig-schuldige sexuelle Annäherung an ein Mädchen. Der Onkel verstösst ihn dann so, wie der Amazonenstaat seine Königin Penthesilea, weil diese sich von individueller Liebe bestricken liess, statt nur auf die Eroberung eines Samenspenders auszugehen. Die Verstoßung durch den Onkel gehorcht dem Besitzdenken im kapitalistischen und, wie zu zeigen sein wird: Elektrisch durchströmten modernsten Amerika. In beiden Fällen aber geht der Verstoßung eine grosse, den strömenden Verlauf der tragischen Handlung aufstauende, lyrisch überhauchte Liebesszene voraus. Im 15. Auftritt bei Kleist

\footnotetext{
${ }^{34}$ Bohemia, 25. September 1912, S. 9. Für die Beschaffung der Bohemia und Tagblatt-Ausgaben (Mikrofilm) sei der Universitätsbibliothek Trondheim an dieser Stelle ganz herzlich gedankt.

${ }^{35}$ Brief vom 9/10. III. 1913. Zitiert nach Franz Kafka: Briefe an Felice, hrsg. v. Erich Heller und Jürgen Born. Frankfurt/Main 1967, S. 332.
} 
und in der („Mozart'schen“) Klavierszene bei Kafka lernen die Helden durch Desillusionierung das Entscheidende: Die Amazonenkönigin begreift, dass Achill sie (und nicht umgekehrt, wie die aus der Ohnmacht Erwachte meinte) besiegt hat. Karl wiederum lernt, dass Klara, während er ihr mozarthaft werbend auf dem Klavier seine kakanische Melodien vorspielt, bereits vergeben ist; an Mack, den Allerobersten des kapitalistischen Machtsystems in Amerika, der sie sogar bereits beschläft. Mack gehören Haus, Frauen und die Zukunft in der Neuen Welt; auch das Haus wird er noch elektrifizieren lassen, womöglich auch deshalb, weil Karl sich im nur von Kerzen erhellten Haus aufführte wie ein zweiter Mozart. Daraus folgt mit tragischer Logik der Absturz beider Helden. Sie werden für immer aus ihrer Bahn geworfen. Beide erwachen aus ihrem romantischen Liebeswahn, der den Kampf zwischen ihnen zunächst nicht nur überstanden, sondern sogar noch vertieft hat. Die Statthalter des Systems machen ihnen die Wahrheit einsichtig, der Über-Kapitalist Mack bei Kafka, Penthesileas Dienerin Prothoe bei Kleist. Die Genannten, die eins sind mit ihrer systemischen Funktion, stechen den träumenden, nur noch verschleiert die Realität entziffernden, von grosser Liebe träumenden Literatur-Helden den Star. Dazu fügt sich ausgezeichnet, dass bei Kafka Karl Rossmann erst wieder auf die „richtige Bahn“ gelangt, als er in der Schlussszene in die Mobilmachung für den Ersten Weltkrieg gerät - wobei der typisch romantische (und eben auch Wienerische: Hofmannsthals Elektra oder Salome!) Zwiespalt zwischen Überzartheit und Brutalität bei Kafka als JiuJitsu-Kampf einer ehemals antik-tragischen Heldin, und als rohes Besitzer-Auftrumpfen von Seiten Macks ironisiert wird.

In beiden Fällen ziehen die für einander bestimmten Liebenden einander „elektrisch“ an. Das ist wörtlich zu nehmen so weit, wie die literarische Praxis der Frühromantik (und dann mehrfach bei E.T.A. Hofmann) reichte: Wenn nämlich zwei Liebende direkt und konkret als „Leydener Flasche“ dargestellt wurden, rabiat jedwede Ästhetik des Schönen verletzend, allein dem betörenden Geheimnis der besonderen Fernwirkung von Elektrizität verpflichtet. Liebe und Elektrizität glichen einander darin, dass beide „alle Hindernisse überwinden“. Während das „Electrische“ in Form von Galvanismus und Mesmerismus bei Kleist noch sehr direkt durch dessen (teilweise persönliche) Nähe zu Mesmer, Ritter und die Frühromantikern gegeben war, wird das Phänomen rund ein Jahrhundert später bei Kafka bereits als, überzeugend realistisch gezeichnetes, eben deshalb anonymes gesellschaftlich-industrielles Ambiente ins Bild geholt. Wie bereits angesprochen: Nicht nur beschäftigen sich Kafkas Tagebücher seitenlang mit dem Erfinder und Geschäftsmann Thomas Alva Edison; auch dessen (und darin impliziert: seines Rivalen Nicolaus Tesla) Anstrengungen, New York von den Niagara-Fällen aus mit Strom zu versorgen, geraten breit und 
kenntnisreich, von beträchtlicher Faszination zeugend, ins Bild. Der junge Kafka, der sich später sogar über die elektrische Ausstattung von HauptstadtCafés kennerische Gedanken machen wird, entwirft seine Neue Welt als eine durchgehend elektrifizierte. Der Verschollene ist, wie gesagt, auch ein Lichtund mithin Elektrizitäts-Roman. Was der Prager Autor nicht zeigt, vermutlich nicht wusste, ist der damalige verbissene Kampf zwischen dem GleichstromBefürworter Thomas Alva Edison und seinem Konkurrenten Nikola Tesla; den letzterer verlor, wiewohl er über das technisch weit überlegene WechselstromPrinzip verfügte. Allerdings enthält die Zeichnung des Onkels viele Züge jener geschäftsmässigen Brutalität, die dem damaligen Prag-Besucher Edison zum Sieg und zur Anwesenheit in Kafkas Tagebuch verholfen hatte. Das vom jungen Kafka bewunderungsvoll-verblüfft gezeichnete Geschäfts-Imperium des Onkels steht in durchgehender Abhängigkeit vom neu eingeführten elektrischen Strom; samt so ziemlich aller Metaphern, die das blitzende, gleissende Phänomen seit alters her um sich zu versammeln gewusst hat. Hans Blumenberg ist wie gesagt der Hauptautor, wenn es um deren Katalogisierung und Aufschlüsselung geht. Kafkas Amerika im Erstling ist jedenfalls eines des künstlichen Lichts (nur selten wird man einer noch "griechischen“ Sonne ansichtig, das geschieht freilich weiterhin passagenweise, ein Reflex noch der Goethe'schen Lichtemphase). Freilich die durchweg ,elektrisch“ betriebene, via Telefon, Telegraf und Telegramm am Laufen gehaltene, hochkomplexe Organisation von Onkel Jakobs Geschäftswelt widerspiegelt einen amerikanischen Kapitalismus in potentia, will sagen: Zeigt Profitgewinn bereits aus der reinen Logistik generiert, und nicht mehr „europäisch“ durch simplen Warenhandel. Diese um das Jahr 1914 herum niedergeschriebenen Darstellungen des ganz modernen, atemlos tätigen, wunderbar profitreichen Kapitalismus aus Kafkas Feder erinnern denn auch, ohne hier leichtfertig ins Aktualisieren zu verfallen, bereits an Amazons Geschäftsmodell. „Das grenzt ja ans Wunderbare“ sagt Karl - und hat Recht. Man war hier um einen strukturellen Quantensprung weiter als im alten Europa, mit dieser „Art Kommissions- und Speditionsgeschäft, wie es, soweit sich Karl erinnern konnte, in Europa vielleicht gar nicht zu finden war.“ „Es war daher ein Geschäft, welches in einem Käufe, Lagerungen, Transporte und Verkäufe riesenhaften Umfangs umfasste und ganz genaue unaufhörliche telefonische und telegraphische Verbindungen mit den Klienten unterhalten musste. Der Saal der Telegraphen war nicht kleiner, sondern grösser als das Telegraphenamt der Vaterstadt ..." Im Telefonsaal: „das Läuten war sinnverwirrend.“ Die elektrische Hölle des neuesten Kapitalismus, aber, notwendigerweise, alles andere als eine dunkle Unterwelt: „Der Onkel öffnete die nächste dieser Türen und man sah dort im sprühenden elektrischen Licht einen Angestellten gleichgültig gegen jedes 
Geräusch der Türe, den Kopf eingespannt in ein Stahlband, das ihm die Hörmuscheln an die Ohren drückte. Der rechte Arm lag auf einem Tischchen, als wäre er besonders schwer und nur die Finger zuckten unmenschlich gleichmässig und rasch." Derart hatten bereits die Froschschenkel unter den galvanischen Versuchen der Mesmeristen gezuckt, ohne dass freilich in ihnen noch wirklich Leben gewesen wäre. Bei Kafka dagegen geht es bereits zu, wie im und um den Atomkern herum in der neuesten Uran-Welt der post-Einstein'schen Physiker: „Mitten durch den Saal war ein beständiger Verkehr von hin und her gejagten Leuten.“ Diese Angestellten gleichen bereits Elementarteilchen auf der Jagd nach ihren Bahnen, sozusagen atemlos in ihrem Bemühen, ganz wie später dann in Michel Houellebecqs epochalem Roman Elementarteilchen. „Keiner grüsste, ...jeder schloss sich den Schritten des vorhergehenden an ... oder fieng mit den Blicken wohl nur einzelne Worte oder Zahlen von Papieren ab, die er in der Hand hielt und die bei seinem Laufschritt flatterten."36 In dieser neu entstandenen Welt der „Angestellten“ im avancierten soziologischen Sinn etwa des Siegfried Kracauer spiegelt sich quasi die Welt der elektromagnetischen Felder und die der entstehenden Quantenphysik wider; und vice versa. Eine Vorwegnahme, womöglich noch viel konkreter als die von Ernst Bloch einst favorisierte „Konkrete Utopie“. Freilich keine „sozialistische“.

\footnotetext{
${ }^{36}$ Sämtliche Zitate: Franz Kafka, Der Verschollene, in: Gesammelte Werke in zwölf Bänden, hrsg. Hans-Gerd Koch, a. a. O., S. 54 f. (s. Anmerkung 38).
} 
Open Access Dieses Kapitel wird unter der Creative Commons Namensnennung 4.0 International Lizenz (http://creativecommons.org/licenses/by/4.0/deed.de) veröffentlicht, welche die Nutzung, Vervielfältigung, Bearbeitung, Verbreitung und Wiedergabe in jeglichem Medium und Format erlaubt, sofern Sie den/die ursprünglichen Autor(en) und die Quelle ordnungsgemäß nennen, einen Link zur Creative Commons Lizenz beifügen und angeben, ob Änderungen vorgenommen wurden.

Die in diesem Kapitel enthaltenen Bilder und sonstiges Drittmaterial unterliegen ebenfalls der genannten Creative Commons Lizenz, sofern sich aus der Abbildungslegende nichts anderes ergibt. Sofern das betreffende Material nicht unter der genannten Creative Commons Lizenz steht und die betreffende Handlung nicht nach gesetzlichen Vorschriften erlaubt ist, ist für die oben aufgeführten Weiterverwendungen des Materials die Einwilligung des jeweiligen Rechteinhabers einzuholen.

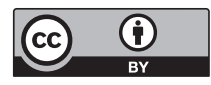

